“C 2016 IEEE. Personal use of this material is permitted. Permission from IEEE must be obtained for all other uses, in any current or future media, including reprinting/republishing this material for advertising or promotional purposes, creating new collective works, for resale or redistribution to servers or lists, or reuse of any copyrighted component of this work in other works." 


\title{
Finding the Optimal Place and Size of an Energy Storage System for the Daily Operation of Microgrids Considering Both Operation Modes Simultaneously
}

\author{
Arash Beiranvand ${ }^{1}$, Mahlagha Mahdavi Aghdam ${ }^{2}$, Student Member, IEEE, Li Li ${ }^{2}$, Member, IEEE, Shouzhen \\ $\mathrm{Zhu}^{3}$, Jinghong Zheng ${ }^{3}$ \\ ${ }^{1}$ Department of electrical engineering, Shahid Beheshti University of Tehran, Tehran, Iran \\ ${ }^{2}$ Centre of Green Energy and Vehicle Innovation, University of Technology Sydney, Ultimo, NSW 2007, Australia \\ ${ }^{3}$ Department of Electrical Engineering, Tsinghua University, Beijing, China \\ Arash.beiranvand65@gmail.com
}

\begin{abstract}
This paper aims to find the optimal place and size of an energy storage system in a microgrid, considering the gridconnected mode and autonomous mode simultaneously. Energy storage systems are one of the most effective components in today's power grids to improve the power quality of power grids, therefore attracting more attention in this field. Specially, in microgirds which use various kinds of distributed generations, using energy storage systems is necessary to improve their power quality. Finding the optimal place and size of energy storage systems is a common action in microgrids. However, it should be noted that most microgrids can be operated in both of their operation modes and finding optimal place and size of an energy storage system for one of these operation modes doesn't mean that they are optimal for the other mode. This paper presents a new method to find the optimal place and size of an energy storage system for microgrids during daily operation, considering both grid-connected mode and autonomous mode simultaneously. The presented method is based on applying the AC-optimal power flow to find the optimal place and size of the energy storage system.
\end{abstract}

Index Terms-Distributed Generation; Energy storage System; Genetic Algorithm; Microgrids;

\section{INTRODUCTION}

$\mathrm{M}$ icrogrids (MGs) are one of the most important components of today's power systems. Most of them have the ability to separate themselves from main power grids during emergencies and continue their operation autonomously [1], [2]. This ability has attracted more attention because various kinds of catastrophic failures occur in main power grids. This feature improves the reliability of power grids drastically [2], [3]. The two operation modes of MGs, that is, islanding mode and grid-connected mode, have been investigated in the literature. For example, in [1], the autonomous operation of MGs is investigated and based on its specific features, a new method to model black out in MGs has been presented.

One of the important features of MGs is that one can find various kinds of distributed Generations (DGs) in their structures. Although applying these DGs has several important advantages and benefits such as economic benefits or environmental advantages, they cause several deficiencies for MGs. One of the most important problems of applying DGs in MGs is that they decrease the quality of the produced power due to a couple of reasons such as the stochastic features of DGs [4-6]. To overcome the problems associated with the power quality of MGs, Energy Storage Systems (ESSs) can be applied. Using ESSs can improve the power quality in power grids drastically and actually they are one of the most effective components in power systems to improve the quality of power. Due to this fact, ESSs have been investigated in the literature. In [7], the author presents a frequency control approach for autonomous MGs by using ESSs to support the frequency of the MG. Also in [8], a novel control algorithm for coordinating a total of 1000 residential electric heat pumps and a battery storage system is presented. The presented method provides MG tie-line smoothing services. For smoothing the tie-line power fluctuations, the suggested method applies two-way communication networks to manage distributed heat pumps in a MG. In [9], the authors address a new technique for the coordination of multiple ESSs to control the voltage in a smart grid.

The most important issue associated with ESSs is finding the optimal places and optimal sizes for them in power grids to improve the power quality. In [10], authors address the optimal energy storage management and sizing problem in the presence of renewable energy and dynamic pricing associated with electricity from the grid. A formulation is set up in [11] to determine the optimal contract capacities and optimal sizes of battery energy storage systems for time-of-use rate customers. Furthermore, in [12] the authors presented a new method for finding the optimal size of ESSs to reduce the operation costs of MGs, using the so-called grey wolf optimization under various constraints.

The problem of these suggested methods is that they aim to find the optimal sizes and places of ESSs in one of the possible operation modes of MGs and distribution systems, 
but as it was mentioned earlier due to a couple of reasons MGs may need to change their operation modes. In the new operation mode the ESSs in those determined places and sizes may be unable to improve the power quality effectively and in some cases they may cause some problems for the operation of MGs and make the condition of grids worse.

This paper presents a new method to find optimal place and size for an energy storage system in MGs, considering the daily operation features of MGs in both of their operation modes simultaneously, that is, grid-connected mode and autonomous mode. The most important difference between the presented method in this paper and other methods is it can find the optimal place and size of the energy storage system in a way that, if the MG changes its operation mode, these size and place are one of the best possible sizes and places for this energy storage in the new operation mode. So, the operation of the MG becomes more reliable and safer. The presented method applies the AC-Optimal Power Flow (AC-OPF) to the MG operation and in this way the presented method becomes more practical. The proposed method is presented in Section II. Section III illustrates a sample network for applying the proposed method. The fitness function and its constraints are defined in Section IV. The efficiency of the presented method is shown in the simulation results in Section V. Finally, Section VI is devoted to the conclusion.

\section{PROPOSED METHOD}

As mentioned, the presented method aims to find optimal places and sizes of ESSs for MGs, considering both operation modes simultaneously. This is the major advantage of the presented method. To find optimal places and sizes of ESSs, Genetic Algorithm (GA) is applied. Also it is assumed that the case study has the fundamental features of MGs and operator is able to control all of the case study components. The following will shed light on the methodology of the presented method and the procedure of applying the GA for finding optimal places and sizes of ESSs.

\section{A. Flowchart of the Proposed Method}

The flowchart of the presented method is shown in Fig.1. In this method, each of chromosomes represents a specific size and place for the ESS. The method of applying the GA for this purpose will be presented in the next part. In the flowchart of the proposed method, $K$ shows the $K^{\text {th }}$ hour of the day.

At the first step, for the first hour of the day, one of the generated chromosomes is selected. Then, for this chromosome the condition of the $\mathrm{MG}$ is updated for both operation modes, that is, islanding mode and grid-connected mode. After this step, for each of these operation modes a specific procedure is applied simultaneously to obtain the relative fitness function of the selected chromosome.

\section{1) Islanding Mode}

For this mode, after updating the condition of the MG, if the amount of the grid loads is larger than the total capacity of power generation, including total capacity of power generation by the DGs plus the capacity of power produced by the ESS, 0.1 p.u. of those loads when the load shedding is allowed, should be shed. This action continues till the total capacity of power production would meet the grid load demand.

At the next step, if the total capacity of power production by the DGs is larger or equal to the total amount of the grid loads plus the size of the ESS and the ESS is able to be charged ( it is not full), the energy storage should be charged. Then, if the energy storage is full, it is not charged.

In the case that the total capacity of power producing by the DGs is less than the total amount of the grid loads, the energy storage should be discharged if it hasn't been discharged thoroughly.

\section{2) Grid-connected Mode}

For this mode, after updating the condition of the MG, if the total capacity of power generation by the DGs is larger or equal to the total amount of the grid loads, the energy storage system could be charged. To charge the energy storage system, it should not be full.

In the case that the total capacity of power generation by the DGs is less than the total amount of the grid loads, if the energy storage system has not be discharged thoroughly, it will be discharged.

After all of these steps, the AC-OPF is calculated. If it is not converged, the relevant chromosome gives a penalty factor and will be deleted from the target population. If the AC-OPF is converged, the obtained information is stored and all of the mentioned steps are repeated for the next hour. This procedure is repeated for all of the day hours and at the end of the day the fitness function for the selected chromosome is calculated. Same procedure is applied for all chromosomes of the target population.

\section{B. Genetic Algorithm}

As it was mentioned, the GA is applied to solve the optimization problem of finding optimal place and size for the energy storage system. To adopt the GA, each of chromosomes of target population is divided into two parts. One part is allocated to find possible places for the energy storage system. Another part is allocated to find possible sizes for the energy storage system. By generating more and more chromosomes, more places and sizes for the energy storage systems are investigated simultaneously. By this way, the presented method could investigate various conditions of the case study to find the optimal solution. For example, consider Table I as one of the target population chromosomes. In this case, the bits between $8^{\text {th }}$ bit to $13^{\text {th }}$ bit are allocated to possible places for energy storage system. The bits are countered from the right side. As a result, this chromosome represents a condition of the case study in which the ESS has connected to bus 8 . Other bits are allocated to find possible sizes for the energy storage system. In this example, the chromosome represents a condition of the case study in which the size of the energy storage system is equal to $75 \mathrm{~kW}$. 


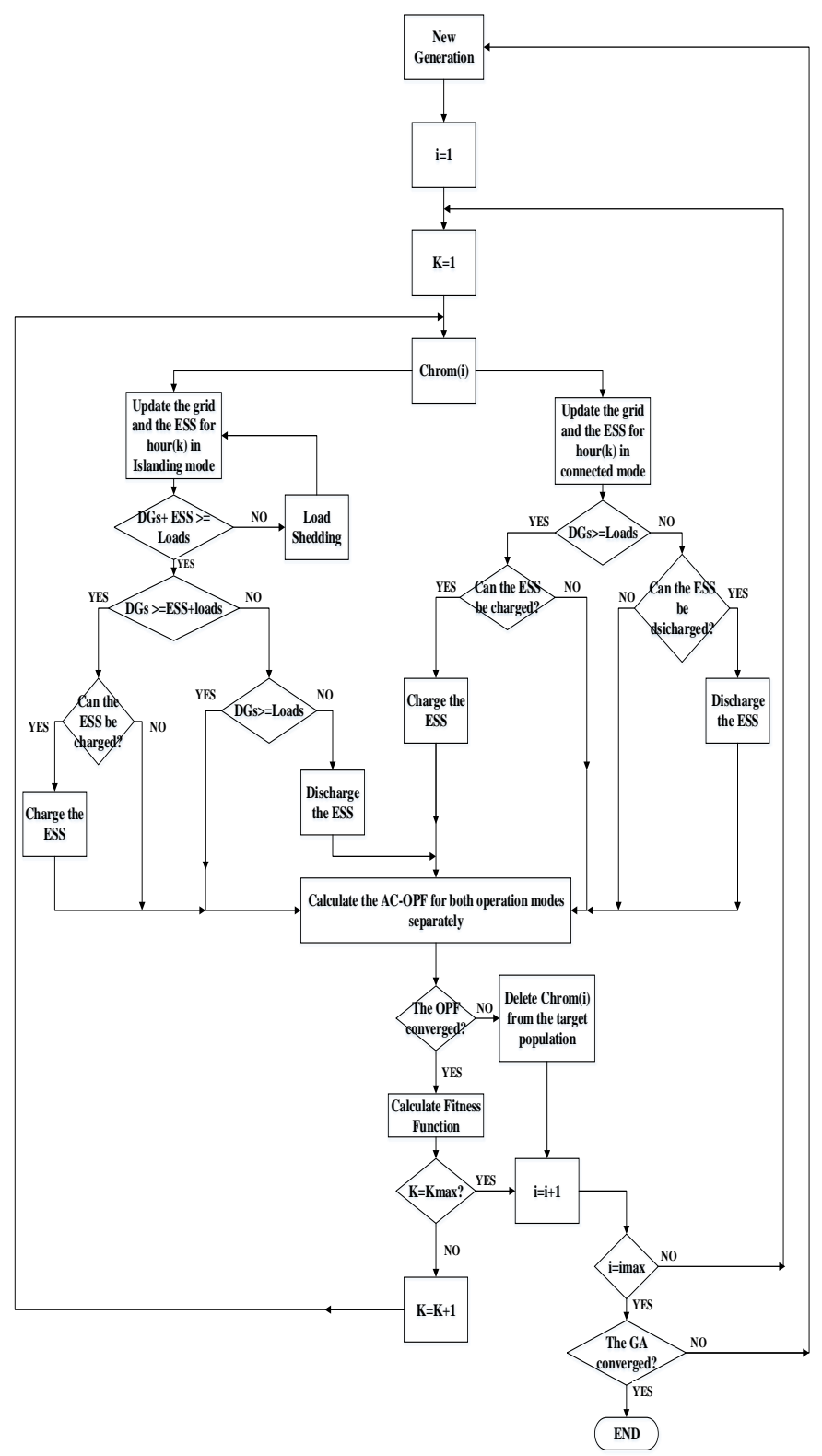

Fig. 1. Flowchart of the proposed method

TABLE I. Sample Chromosome

\begin{tabular}{|l|l|l|l|l|l|l|l|l|l|l|l|l|}
\hline 1 & 0 & 0 & 1 & 0 & 1 & 1 & 0 & 0 & 1 & 0 & 0 & 0 \\
\hline
\end{tabular}

\section{SAMPLE NETWORK}

The IEEE 33-bus standard grid is selected for the sample grid of this paper. The detailed information of this grid could be found in the appendix, Table IV, and [9]. It has been assumed that this grid has two DGs at its bus 9 and bus 30 . The DG at bus 9 includes 3 diesel generators and the DG at bus 30 includes 6 Doubly Fed Induction Generator (DFIG) wind turbines. The details of these generators are shown in Table III in the appendix. Also it is assumed that the loads 9, 18,30 are important loads and the load shedding is not allowed for them. Fig. 2 depicts this case study.
Fig. 3 shows the average of power in p.u. obtained from the wind power plant during various periods of the operation day. The load profile of the case study is shown in Fig. 4 for various periods of the day.

As it was mentioned earlier, the presented method aims to find the optimal place and size for the ESS, considering both autonomous and grid-connected modes simultaneously. The specific conditions of the case study for these operation modes are as follow,

\section{A. Grid-connected Mode}

In this mode, the radial structure of the case study in Fig. 2 is kept and the case study is connected to the main power grid through bus 1 which is considered as the slack bus for solving the AC-OPF.

\section{B. Islanding Mode}

Keeping the radial structure of the MGs in this mode is not safe, and in this mode it is tried to make exactly one loop in the MG structure so that the maximum numbers of DGs would be connected with each other through this loop [1], [2]. Based on this fact, the presented structure in Fig.5 is selected for this mode. Also, in this mode, bus 9 is considered as the slack bus of the case study for solving the AC-OPF.

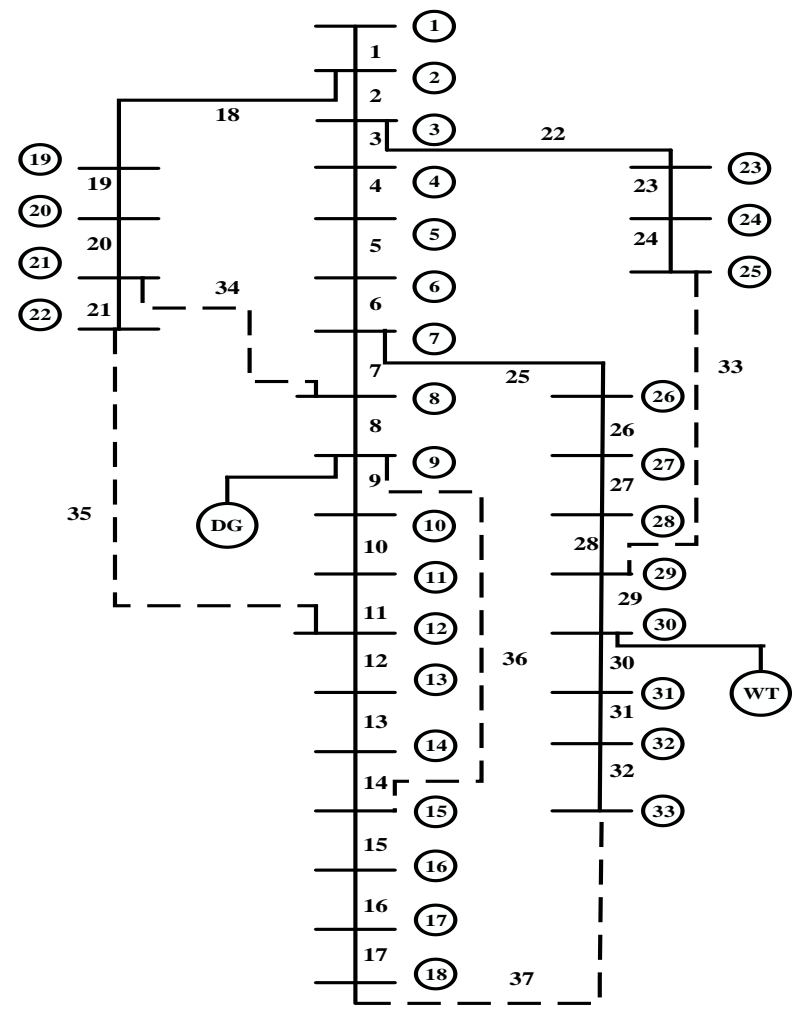

Fig. 2. IEEE 33-bus standard grid 


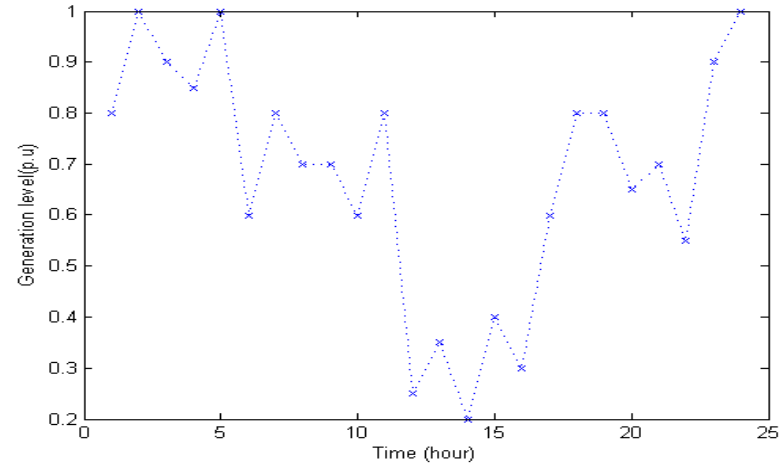

Fig. 3. Wind turbines generation during the day

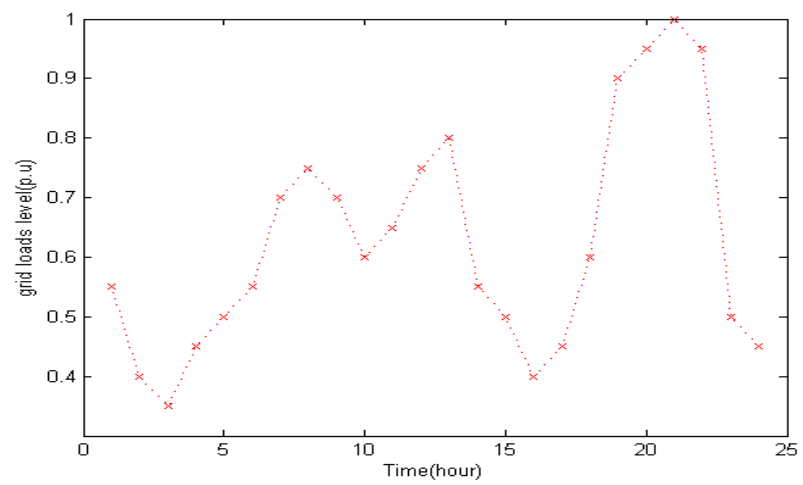

Fig. 4. Grid loads level during the day

\section{THE FITNESS FUNCTION}

Aforementioned, the presented method uses the results of calculating the AC-OPF to obtain relevant fitness functions for each of the target population chromosomes.

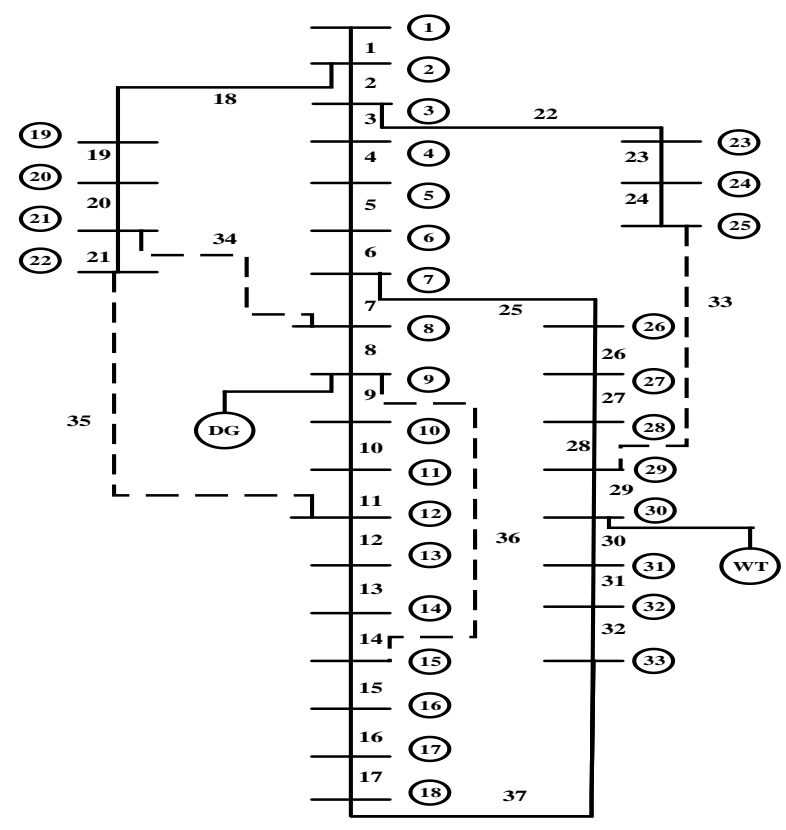

Fig. 5. The suggested structure for the autonomies mode
It is assumed that the MG operator can only dispatch the DGs of the grid, including the wind power plant and the diesel generator power plant. Also, the costs associated with the annual substitution of the ESS and the primary cost of engaging the ESS has not been considered. For calculating the AC-OPF for the case study, the relevant cost functions for each of the DG units should be determined. The cost function for the wind power plant is as follow:

$$
F_{\text {wind }}=\sum_{i=1}^{N_{w}} \beta_{i} P_{\text {wind }, i}
$$

where $N_{w}$ is the number of wind turbines in the wind power plant. Also, the cost function for the diesel generator power plant is as follow,

$$
F_{\text {Diesel }}=\sum_{i=1}^{N_{d}}\left(\alpha_{i}+\beta_{i} P_{\text {Diesel }, i}+\gamma_{i} P_{\text {Diesel }, i}^{2}\right)
$$

where $N_{d}$ is the number of diesel generators in the diesel generator power plant. The data of these DG's can be found in the Table I In the appendix. So, the total cost function for the case study is:

$$
F_{\text {Cost }}=F_{\text {Wind }}+F_{\text {Diesel }}
$$

To solve the AC-OPF, a couple of constraints are considered. The constraint on the produced reactive power by DGs is defined as:

$$
Q_{G, i}^{M i n} \leq Q_{G, i} \leq Q_{G, i}^{M a x}
$$

where $Q_{G, i}^{M i n}$ and $Q_{G, i}^{\operatorname{Max}}$ are the maximum and minimum amount of the produced reactive power by DG $(i)$. Then, the constraint on the produced active power by DGs is:

$$
P_{G, i}^{\operatorname{Min}} \leq P_{G, i} \leq P_{G, i}^{\operatorname{Max}}
$$

where $P_{G, i}^{M i n}$ and $P_{G, i}^{M a x}$ are the maximum and minimum amount of the produced active power by DG $(i)$. Voltage constraint for the bus voltages is defined as:

$$
V_{i}^{\text {Min }} \leq V_{i} \leq V_{i}^{\text {Max }}
$$

where $V_{i}$ is the voltage of the bus $(i)$. The maximum and minimum amount of the bus voltages are 1.05 p.u. and 0.95 p.u. respectively. The loading of the grid lines is limited to:

$$
L_{i}^{M i n} \leq L_{i} \leq L_{i}^{M a x}
$$

where $L_{i}$ is the loading of the line $(i)$. The obtained result should satisfy the power flow equation as follows:

$$
S_{i}=P_{i}+j Q_{i}=V_{i}\left[\sum_{j=1}^{n} Y_{i j} V_{j}\right]^{*}
$$

where $S_{i}$ is the apparent power injected to bus (i). 
Based on the obtained results from calculating the AC$\mathrm{OPF}$, the fitness function is obtained from the following equation:

$F F=\min [\underbrace{\left(\sum_{j=1}^{24} \sum_{i=1}^{33}\left|1-V_{i}^{j}\right|\right)+\left(\sum_{j=1}^{24} \sum_{i=1}^{37}\left(P_{i}^{L \text { Loss }}\right)_{j}\right)}_{\text {connected }}+\underbrace{\left(\sum_{j=1}^{24} \sum_{i=1}^{33}\left|1-V_{i}^{j}\right|\right)+\left(\sum_{j=1}^{24} \sum_{i=1}^{37}\left(P_{i}^{\text {Loss }}\right)_{j}\right)}_{\text {Islanding }}]$

In this equation, $V_{i}^{j}$ is the voltage of bus $(i)$ for hour $(j)$ of the day and $\left(P_{i}^{\text {Loss }}\right)_{j}$ is total power loss of line $(i)$ for hour $(j)$ of the day. This presented fitness function considers both autonomous mode and grid-connected mode simultaneously. It should be noted that, to obtain the total grid losses, at first, $R I^{2}$ is calculated for each MG line. The sum of all $R I^{2}$ in a grid represents the total losses of the MG.

\section{Simulation Results}

As it was mentioned, the presented method uses the GA to solve the optimization problem. For this purpose, it is assumed that each chromosome has 13 bits so that, the first seven bits from the left side are allocated to find the optimal size of the energy storage system and other bits are allocated to find the optimal place for it. Also, it is assumed that the maximum size of the energy storage system could be half of the size of the biggest DG in the case study and the charge/discharge rate of the energy storage is equal to [size/4] $\mathrm{kWh}$. To simulate the presented method, MATLAB and MATPOWER are applied.

The relevant chromosome of the optimal solution which is obtained from the GA, can be seen in Table 2. This chromosome shows that the optimal size for this energy storage system is equal to $101 \mathrm{~kW}$ and the optimal place for the energy storage is bus 25 .

TABLE II. The optimal solution

\begin{tabular}{|l|l|l|l|l|l|l|l|l|l|l|l|l|}
1 & 0 & 1 & 1 & 0 & 1 & 1 & 0 & 1 & 1 & 0 & 0 & 1 \\
\hline
\end{tabular}

Fig. 6 shows a comparison between the daily average bus voltages before and after applying the energy storage system for both operation modes.

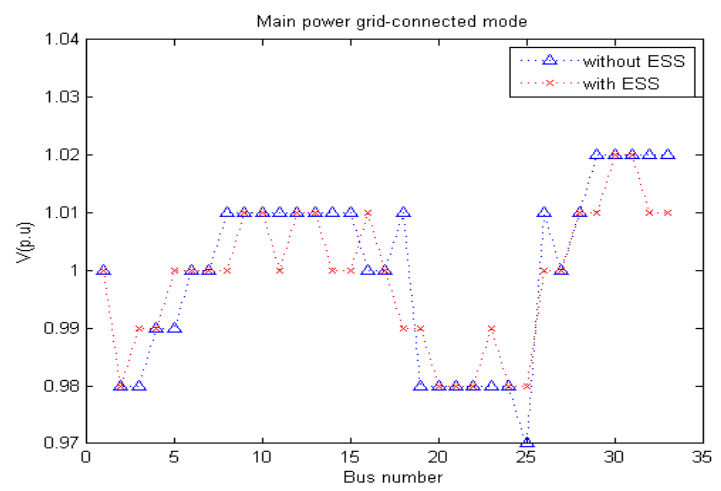

a) Grid-connected mode

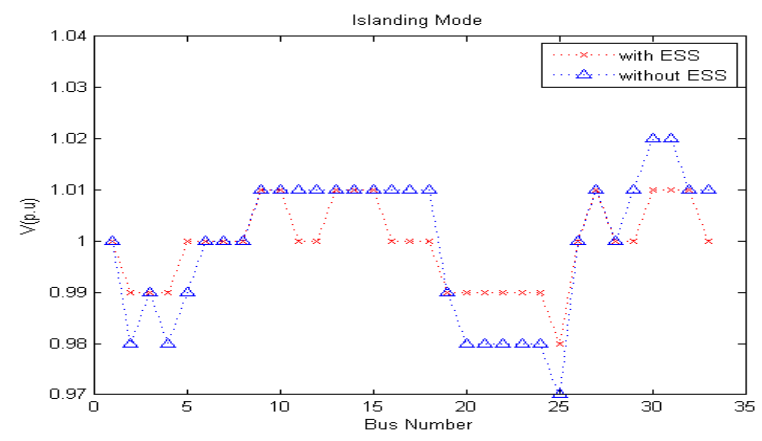

b) Islanding mode

Fig. 6. Daily average voltages before and after applying the energy storage system a) Grid-connected mode b) Islanded mode

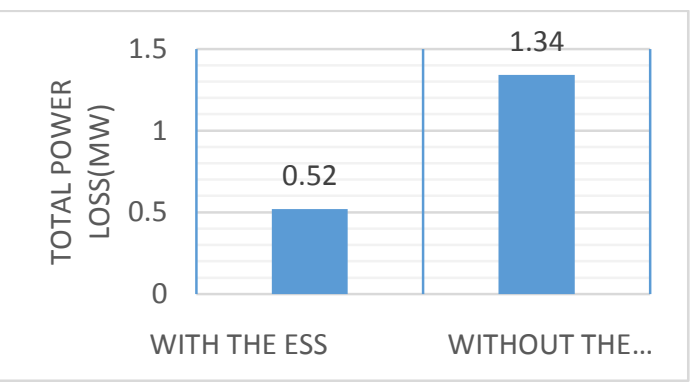

a) Grid-connected mode

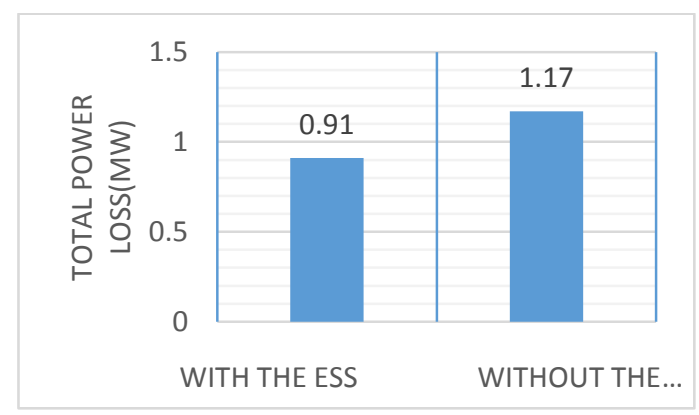

b) Islanding mode

Fig. 7. Grid power loss for both operation modes with and without the ESS a) Grid-connected mode b) Islanded mode

Fig. 7 shows a comparison between the power loss before and after applying the energy storage system for both operation modes.

As it is clear, the presented method has the bus voltages in both operation modes of the MG closest to 1 p.u. and also it reduces the total power loss of the MG in both operation modes. As a result, the obtained results show the efficiency of the presented method.

\section{CONCLUSION}

This paper presents a new method to find the optimal place and size of energy storage for the daily operation of MGs. The most important advantage of the presented method in compare with other methods in this field is that it considers both operation modes of MGs simultaneously during finding the 
optimal place and size of the energy storage system. Due to this feature if MGs change their operation modes, in the new operation modes the energy storage systems are still in appropriate places and have appropriate sizes. As a result they could be applied in these new operation modes properly. The presented method applies genetic algorithm to solve the optimization problem of finding the optimal place and size of the energy storage system. Also, the presented method uses the results of calculating the AC-OPF for calculating the fitness function of the GA in this method. The obtained results from simulating the presented method show that the presented method is effective in both improving voltage profile and decreasing total grid power loss.

\section{APPENDIX}

The details of the generators connected to Bus 9 and 30 are tabulated in Table III. The line data of IEEE 33-bus standard grid is listed in Table IV.

Table III. The wind and diesel generator data used in the example microgrid

\begin{tabular}{|l|c|c|}
\hline Generator Type & Diesel & Wind \\
\hline$\alpha(\$ / h)$ & 0.2731 & 0 \\
\hline$\beta(\$ / k W h)$ & 0.1453 & 0.032 \\
\hline$\gamma\left(\$ /(k W)^{2} h\right)$ & 0.0042 & 0 \\
\hline$P_{\min }(k W)$ & 0 & 0 \\
\hline$P_{\max }(k W)$ & 800 & 300 \\
\hline $\cos \varphi$ & 0.6 & 0.8 \\
\hline
\end{tabular}

Table IV. The line data of IEEE 33-bus grid

\begin{tabular}{|c|c|c|c|c|c|c|c|}
\hline $\begin{array}{c}\text { From } \\
\text { bus }\end{array}$ & $\begin{array}{c}\text { To } \\
\text { bus }\end{array}$ & R(p.u.) & X(p.u.) & $\begin{array}{c}\text { From } \\
\text { bus }\end{array}$ & $\begin{array}{c}\text { To } \\
\text { bus }\end{array}$ & R(p.u.) & X(p.u.) \\
\hline 2 & 3 & 0.03076 & 0.01567 & 2 & 19 & 0.01023 & 0.00976 \\
\hline 3 & 4 & 0.02284 & 0.01163 & 19 & 20 & 0.09385 & 0.08457 \\
\hline 4 & 5 & 0.02387 & 0.01211 & 20 & 21 & 0.02555 & 0.02985 \\
\hline 5 & 6 & 0.0511 & 0.04411 & 21 & 22 & 0.04423 & 0.05848 \\
\hline 6 & 7 & 0.01168 & 0.03861 & 3 & 23 & 0.02815 & 0.01924 \\
\hline 7 & 8 & 0.04439 & 0.01467 & 23 & 24 & 0.05603 & 0.04424 \\
\hline 8 & 9 & 0.06426 & 0.04617 & 24 & 25 & 0.0559 & 0.04374 \\
\hline 9 & 10 & 0.06514 & 0.04617 & 6 & 26 & 0.01267 & 0.00645 \\
\hline 10 & 11 & 0.01227 & 0.00406 & 26 & 27 & 0.01773 & 0.00903 \\
\hline 11 & 12 & 0.02336 & 0.00772 & 27 & 28 & 0.06607 & 0.05826 \\
\hline 12 & 13 & 0.09159 & 0.07206 & 28 & 29 & 0.05018 & 0.04371 \\
\hline 13 & 14 & 0.03379 & 0.04448 & 29 & 30 & 0.03166 & 0.01613 \\
\hline 14 & 15 & 0.03687 & 0.03282 & 30 & 31 & 0.0608 & 0.06008 \\
\hline 15 & 16 & 0.04656 & 0.034 & 31 & 32 & 0.01937 & 0.02258 \\
\hline 16 & 17 & 0.08042 & 0.10738 & 32 & 33 & 0.02128 & 0.03319 \\
\hline 17 & 18 & 0.04567 & 0.03581 & 1 & 2 & 0.00575 & 0.00293 \\
\hline
\end{tabular}

\section{REFERENCES}

[1] M. Khederzadeh, A. Beiranvand, "Identification and Prevention of Cascading Failures in Autonomous Microgrid", IEEE SYSTEMS JOURNAL, Oct, 16. 2015, DOI: 10.1109/JSYST.2015.2482227

[2] E. Sortomme, S. S. Venkata, and J. Mitra, "Microgrid protection using communication-assisted digital relays," IEEE Trans. Power Del., vol. 25, no. 4, pp. 2789-2796, Oct. 2010.

[3] P. Tenti, H. K. M. Paredes, F. P. Marafão, P. Mattavelli, "Accountability in Smart Microgrids Based on Conservative Power Theory", IEEE Trans. on Instrumentation and Measurement, Sept. 2011, vol. 60, no. 9.

[4] M. Basu and A. Chowdhury, "Cuckoo search algorithm for economic dispatch," Energy, vol. 60, pp. 99-108, Oct. 2013.

[5] B. K. Kang; S. T. Kim; B. C. Sung; J. W. Park, "A Study on Optimal Sizing of Superconducting Magnetic Energy Storage in Distribution Power System", IEEE Transactions on Applied Superconductivity. NOV, 03, 2011. Vol 2, Issue 3.

[6] L. Meegahapola, E. Vittal, A. Keane, and D. Flynn, "Voltage security constrained reactive power optimization incorporating wind generation," in Power System Technology (POWERCON), 2012 IEEE International Conference on, 2012, pp. 1-6.

[7]

[8] Serban, C. Marinescu, "Control Strategy of Three-Phase Battery Energy Storage Systems for Frequency Support in Microgrids and with Uninterrupted Supply of Local Loads", IEEE Transactions on Power Electronics, vol. 29, issue. 9, pp. 5010-5020, Sep. 24. 2013.

[9] D. Wang, S. Ge, H. Jia, C. Wang, "A Demand Response and Battery Storage Coordination Algorithm for Providing Microgrid Tie-Line Smoothing Services", IEEE Transactions on Sustainable Energy, vol. 5, issue. 2, pp. 476-486, Jan. 21. 2014

[10] L. Wang, D. H. Liang, A. F. Crossland, P.C Taylor, "Coordination of Multiple Energy Storage Units in a Low-Voltage Distribution Network", IEEE Transactions on Smart Grid, vol. 6, issue. 6, pp. 2906-2918, July. 29. 2015

[11] P. Harsha ; M. DahlehOptimal, "Optimal Management and Sizing of Energy Storage Under Dynamic Pricing for the Efficient Integration of Renewable Energy", IEEE Transactions on Power Systems Aug, 19, 2014. Vol. 30, Issue 3.

[12] T. Y. Lee, N. Chen, "Determination of optimal contract capacities and optimal sizes of battery energy storage systems for time-of-use rates industrial customers", IEEE Transactions on Energy Conversion, Aug. 6. 2002, pp: 562-568.

[13] S. Sharma, S. Bhattacharjee, A. Bhattacharya, "Grey wolf optimization for optimal sizing of battery energy storage device to minimize operation cost of microgrid", IET Generation, Transmission \& Distribution, vol. 10, issue. 3, pp. 625-637, March. 03. 2016

[14] E. Afzalan, M. A. Taghikhani, M. Sedighizadeh, "Optimal Placement and Sizing of DG in Radial Distribution Networks Using SFLA", International Journal of Energy Engineering, 2012, 2(3): 73-77. 